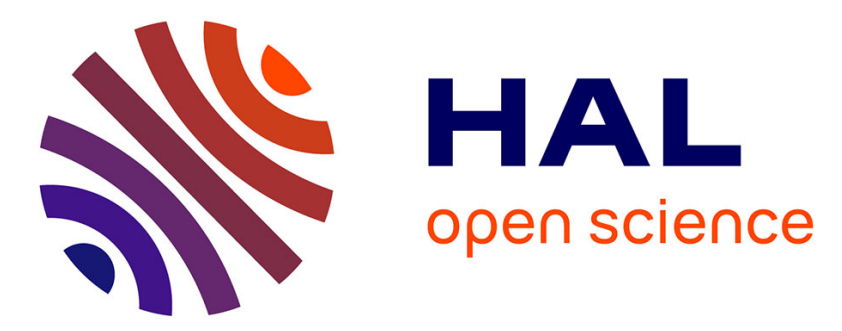

\title{
Editorial: 23rd and 24th Meetings of the French Society of Toxinology (SFET): Special issue on "Toxins: Immunity, Inflammation and Pain"
}

Evelyne Benoit, Julien Barbier, Jordi Molgó

\section{- To cite this version:}

Evelyne Benoit, Julien Barbier, Jordi Molgó. Editorial : 23rd and 24th Meetings of the French Society of Toxinology (SFET): Special issue on "Toxins: Immunity, Inflammation and Pain". Toxicon, 2018, 149, pp.1 - 5. 10.1016/j.toxicon.2018.04.020 . hal-01828958

\section{HAL Id: hal-01828958 \\ https://hal.science/hal-01828958}

Submitted on 20 May 2020

HAL is a multi-disciplinary open access archive for the deposit and dissemination of scientific research documents, whether they are published or not. The documents may come from teaching and research institutions in France or abroad, or from public or private research centers.
L'archive ouverte pluridisciplinaire HAL, est destinée au dépôt et à la diffusion de documents scientifiques de niveau recherche, publiés ou non, émanant des établissements d'enseignement et de recherche français ou étrangers, des laboratoires publics ou privés. 
Editorial

$23^{\text {rd }}$ and $24^{\text {th }}$ Meetings of the French Society of Toxinology (SFET) Special Issue on "Toxins: Immunity, Inflammation and Pain"

The $23^{\text {rd }}$ and $24^{\text {th }}$ Meetings of the French Society of Toxinology (SFET) were held at the Pasteur Institute in Paris on December $5^{\text {th }}-6^{\text {th }} 2016$ and December $7^{\text {th }}-8^{\text {th }} 2017$, with the main subjects being "Toxins: Immunity, Inflammation and Pain" and "Toxins: Biodiversity, Environment and Evolution", respectively. Each meeting gathered about 100 participants from Algeria, Belgium, Benin, China, France, Germany, Italy, Israel, Lebanon, Morocco, Mexico, Republic of Congo, Slovenia, Spain, Switzerland, Tunisia, United Kingdom, and the United States of America. The percentage of foreigner attendees doubled between 2016 and 2017. For each meeting, the SFET aimed to maintain a fair balance among participants interested in toxins from the animal kingdom and those from other sources including bacteria, plants and fungi (see below).

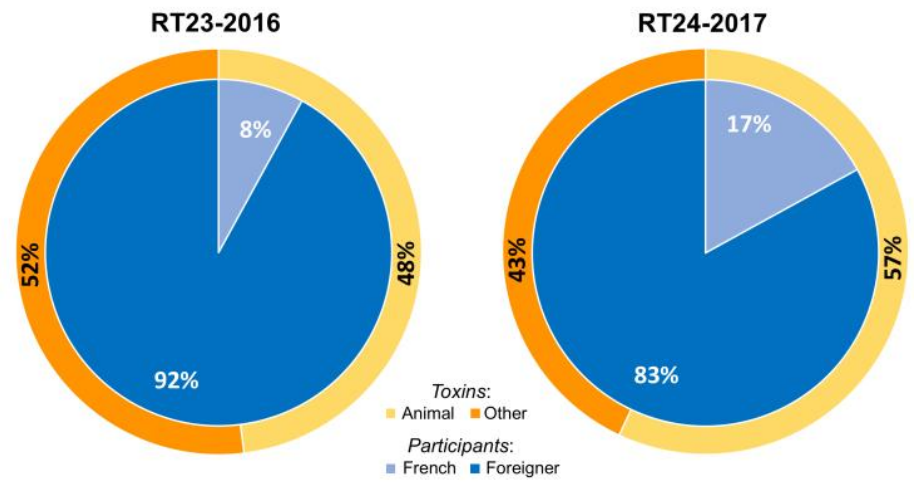

Each meeting included three thematic sessions, during which seven and thirteen speakers were invited as international experts in the field. Additional researchers and students (French or foreign) exposed their work through oral communications and posters. Moreover, each meeting ended with a miscellaneous session that aimed to emphasise the latest research findings in the toxinology area.

We warmly thank all those who made the $23^{\text {rd }}$ and $24^{\text {th }}$ SFET Meetings on Toxinology achievable, and we also acknowledge the contribution of Professor Glenn King, Editor-in-Chief of Toxicon, for his help in the publication of this proceedings as a Special Issue focused on "Toxins: Immunity, Inflammation and Pain" and gathering peer-reviewed original articles and reviews. This Special Issue also includes the abstracts reflecting the oral communications and posters presented during the two SFET meetings. This editorial will summarize the research findings presented as oral communications during the two meetings.

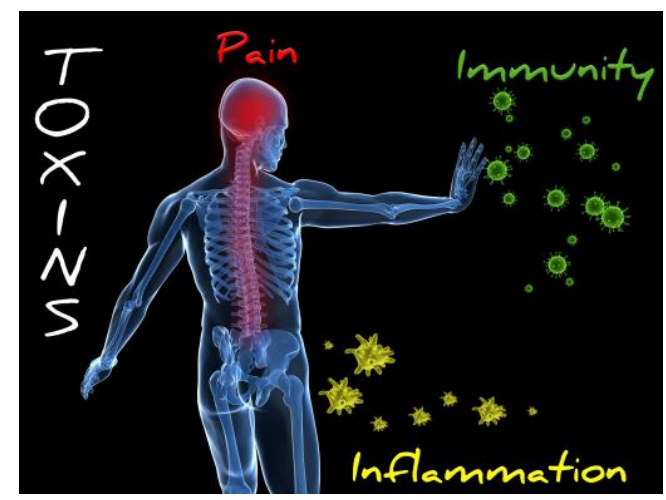

The first session of the $23^{\text {rd }}$ SFET Meeting on Toxinology was devoted to Toxins and Immunity. During an infection by a microorganism, the human body triggers both innate and adaptive immune responses to get rid of the pathogen by both humoral and cell-mediated components. The immune system toolbox encompasses basic mechanisms, such as phagocytosis, antimicrobial peptides called defensins, and the complement system, to more sophisticated defense mechanisms like 
immunological memory. Some bacterial toxins released during host invasion contribute considerably to the outcomes of the infection since they disrupt the host immune cell function. Speakers addressed this host-pathogen interaction field of research in this session. Lhousseine TOUQUI (Paris, France) reported the identification of toxins and pathogen-associated molecular patterns (PAMPs) of Pseudomonas aeruginosa and Staphylococcus aureus involved in airways infection, which is a major problem in several lung diseases. Moreover, the role of PAMPs and of their pattern-recognition receptors (PRRs, expressed by cells of the innate immune system) in inflammation have been described. Finally, mechanisms of expression and the bactericidal effects of mediators, such as cathelicidin and PLA2 antimicrobial peptides produced by host cells, were illustrated both in vitro and in vivo. Nassim MAHTAL (Gif-sur-Yvette, France) performed a highthroughput screening to identify Rac1 immunomodulators able to stimulate innate and acquired immunity. For this purpose, Cytotoxic Necrotizing Factor 1 (CNF1) from uropathogenic strains of Escherichia coli, known to possess efficient immune-stimulatory properties, was used to activate the small GTP-binding protein Rac1 leading to its degradation by the proteasome. From thousands of chemical compounds tested, thirty-five hit molecules were competent to lower degradation of the active form of Rac1 in CNF1-treated human umbilical vein endothelial cells, paving the way for the rational design of new immunostimulating drugs.

However, modifying immune cell behavior is not a feature inherent only to bacterial toxins. Toxins produced by dinoflagellates and cyanobacteria have increasingly become a public health concern for humans. Marine phycotoxins, such as ciguatoxins and brevetoxins, are responsible for human poisonings. The ability of these cyclic polyether toxins to target neuronal cells is well established, leading to a persistent activation of voltage-gated sodium channels, blockade of voltage-gated potassium channels, and increased neuronal excitability. Yet, beyond their canonical action on the nervous system, some studies underlined auxiliary effects of these toxins on the host immune system. This lesser-known property of marine phycotoxins is reviewed in detail by $\mathbf{O}$. PIERRE $\boldsymbol{e t}$ al. in this issue. Furthermore, freshwater cyanobacteria (blue-green algae) produce many biologically active peptides, some of which are potentially harmful for humans. Along with hepatotoxic and tumor-promotional potency, microcystin-LR (MC-LR) is known to impact macrophages involved in innate immunity. Yet, this immunomodulary effect monitored by cytokine production by macrophages is not observed for aerugenosin Aer-865 and two microcystin variants (MC-YR and MC-RR) that differ from MC-LR by only a single amino acid (Z. MOOSOVA et al., in this issue). These structure-dependent effects support the hypothesis of a cell activation triggered by specific surface receptors, and also emphasize this largely unexplored field of research on cyanobacterial metabolites.

Defensins are one of the best-characterized groups of host-defense peptides against pathogens. They are small cysteine-rich cationic proteins highly diverse in their sequences and structures, originating from both the plant and animal kingdoms. Their cysteine motif and disulfide connectivity result in different conformations that drive their classification. Philippe BULET (Saint-Julien-en-Genevois, France) gave an exciting report about toxins found in venomous vertebrates (platypus, snakes, lizards) and arachnids (scorpions, spiders) closely related to defensins. By understanding their structure-activity relationship, these toxins may represent unexpected immune defense therapeutics.

The adaptive immune response can be either humoral or cell-mediated. The humoral response is mediated by B lymphocytes which release antibodies specific to the infectious agent. In the case of the fast intoxination by venomous animals, the body's immune system is unable to produce 
antibodies to antagonise toxins quickly enough. Thus, passive immunity based on the administration of immunoglobulins or sera, produced by an animal that has been previously hyperimmunized against the venom of the corresponding or a very close species, is then commonly used to fight effects of the venom. Although animal-derived antisera remain the cornerstone of venom therapy, biotechnological advances are driving the emergence of different antivenom formats based on human or camelid antibody scaffolds. Fatima CHGOURY (Casablanca, Morocco) reported the generation of nanobodies (VHH antibodies) by phage display directed against the two major neurotoxins of the scorpion Androctonus australis hector and demonstrated the capacity of these nanobodies to cross-react and neutralize a toxic fraction of Androctonus mauretanicus venom. This work highlighted the promising potential of nanotherapeutics for treating scorpion envenoming in North Africa.

Moreover, there are still weaknesses associated with the use of antibody-based serotherapy. Improving the efficacy of envenomation treatments depends on knowledge of the venom's content, because only a limited number of neurotoxins are responsible for its lethality. Thus, it is essential to identify in any venom the most active toxins for each antigenic group, in order to develop the best processes of neutralizing them. To this aim, B.L. EMERICH et al. established in this issue venomic profiles of four Buthus occitanus subspecies, and gave new insights for improving immunotherapy for envenoming by buthid scoprions. Besides antibody-based therapy, innovative strategies emerged aiming to impair the action of neurotoxins. Thus, Michel DE WAARD (Nantes, France) presented a selection of single strand DNA oligonucleotide aptamers directed against the $\alpha-\operatorname{PrXA}$, a conotoxin from the marine cone snail Conus parius that targets nicotinic acetylcholine receptors. Strikingly, these aptamers were active at sub-micromolar concentrations not only in vitro, but also in vivo even if administered after toxin exposure. The role of immune cells in rheumatoid arthritis (RA) is well established since fibrobast-like synoviocytes (FLS) that produce lubrification for the joints become highly invasive during RA. Moreover, pathogenic phenotypes of FLS, compared to healthy cells, overexpress $\mathrm{K}_{\mathrm{Ca}} 1.1$ potassium channels at the plasma membrane. Christine BEETON (Houston, USA) investigated the efficacy of the $\mathrm{K}_{\mathrm{Ca}} 1.1$ inhibitor iberiotoxin from the venom of the scorpion Buthus tamulus, demonstrating in vivo a decrease of the disease severity as well as reduced side-effects compared to the use of the paxilline mycotoxin.

The second session Toxins and Pain started with a keynote presentation by Laurence BOURGEAIS RAMBUR (Paris, France) in which she gave an overview of the physiological mechanisms of pain and the important pain pathways. She clearly described how noxious stimuli can activate peripheral primary afferent sensory fibres, how messages are forwarded to the spinal cord, and then through numerous ascending pathways to encephalic structures, becoming, in fine, a painful sensation. Furthermore, she depicted endogenous modulation mechanisms that act in concert with the ascending pathways. Ryul SONG (Lille, France) presented convincing evidence on the mechanisms involved in the extensive painless lesions accompanying a severe infectious skin disease known as Buruli ulcers caused by Mycobacterium ulcerans. She explained that absence of pain in Buruli ulcer disease cannot be attributed to nerve damage, but involves the action of the mycolactone toxin in a specific cellular pathway targeting $\mathrm{AT}_{2}$ (angiotensin II type 2) receptors, leading to potassium-dependent hyperpolarization of dorsal root ganglia (DRG) sensory neurons.

There is growing clinical evidence suggesting that botulinum neurotoxin (BoNT) type-A may be useful in the treatment of pain disorders, and that it may interfere with pain processing. Mikhail KALINICHEV (Les Ulis, France) presented detailed information on the action of abobotulinumtoxinA (aboBoNT-A) on a rat model of paclitaxel-induced polyneuropathy. In this 
model, aboBoNT-A produced analgesia not only in the injected paw, but also in the contralateral (non injected) paw. In other rat models of chronic pain (diabetic polyneuropathy), further evidence was obtained on the bilateral analgesic effects of aboBoNT-A. The possible mechanisms involved in the bilateral analgesic effects of aboBoNT-A (following unilateral administration) were discussed, and it was suggested that they may involve retrograde axonal transport of the toxin into the spinal cord. The purification of a new potent neuropeptide, named BotAF, from the scorpion Buthus occitanus tunetanus is reported by R. MAATOUG et al. in this issue. BotAF is a 64residue long-chain peptide that shares high similarity with $\beta$-like scorpion toxins. It efficiently abolished acute and inflammatory (both somatic and visceral) pain in rodent models, and did not exhibit toxicity or motor impairment. BotAF fails to reduce inflammatory edema, and its activity is not affected by naloxone. Dose-dependently, the peptide inhibited lumbar spinal cord c-fos/c-jun mRNA up regulation. Altogether, the data presented favour a spinal or peripheral anti-nociceptive mode of action for BotAF.

Tânia C. GONÇALVES (Vitry-sur-Seine, France) presented the first evidence for a new potential antinociceptive agent following the high-throughput screening of a Sanofi collection of venom toxins, using automated patch-clamp recordings on HEK cells overexpressing human $\mathrm{Na}_{\mathrm{v}}$ subtypes. The so-called D peptide shared the same inhibitory cystine knot (ICK) motif with toxins previously reported from the same theraphosid spider family. In mouse DRG neurons, the peptide inhibited tetrodotoxin (TTX)-sensitive Na channels, mainly the $\mathrm{Na}_{\mathrm{v}} 1.7$ subtype, and exhibited lower affinity for TTX-resistant $\mathrm{Na}_{\mathrm{v}} 1.8$ and $\mathrm{Na}_{\mathrm{v}} 1.9$ channels. Furthermore, the peptide had no evident effects on the mouse neuromuscular system in vivo.

Acid-sensing ion channels (ASICs) are trimeric, voltage-independent cation channels activated by extracellular acidification and expressed throughout the vertebrate central and peripheral nervous systems. Eric LINGUEGLIA (Valbonne Sophia Antipolis, France) in his presentation reviewed the pathophysiological roles of ASICs in pain combining different experimental approaches. Among the original approaches was the use of toxins obtained from animal venoms that specifically target ASICs. With this aim, he emphasized the work that has been performed with mambalgins (from mamba venom), which are gating modifiers, used to reveal the role of subtypes of ASICs in pain, including inflammatory and neuropathic pain. The resolved 3D crystal structure of mambalgin-1, allowed delineation of the pharmacophore for ASIC inhibition and further peptide optimization.

The third session was dedicated to Toxins and Inflammation. Although host cells are regularly in contact with infectious agents, the microbes hardly provoke disease. This is due to protection of the mucosal tissues by the innate immune system. Inflammatory processes are activated as a result of complex interactions between pathogens and host target cells. Albeit inflammation orchestrated by cytokines is necessary for eliminating microbes, it may also be detrimental to the host and is consequently a highly regulated process. Jean-Marc CAVAILLON (Paris, France) gave a keynote presentation on how bacterial exotoxins (superantigens, pore-forming toxins), and endotoxins (lipopolysaccharides, LPS) induce a potent activation of inflammatory or anti-inflammatory cytokines (see the review article by J-M. CAVAILLON in this issue). Moreover, Félix VIANA (San Juan de Alicante, Spain) highlighted the identification of TRPA1, a non-selective cation channel with a critical role in chemo-nociception, as a sensor for LPS endotoxin. This work demonstrated that TRPA1 channels are key molecular determinants of rapid LPS effects on sensory neurons leading to acute neurogenic inflammation in vitro and in vivo. Results also suggested that TRPA1 channels may play a role in the first line of immune defense against microbes. 
Another novel investigation led by Jean-David MOREL (Paris, France) questioned why infected people fail to mount protective immune responses against Mycobacterium ulcerans responsible for Buruli ulcer disease. Actually, mycolactone, a polyketide toxin produced by $M$. ulcerans, diffuses in infected hosts and, by targeting the host receptor Sec61 translocon, dampens the cellular immune response. This work illustrated the potential of mycolactone for tuning inflammatory response. Clostridium sordellii and Clostridium difficile are two closely related microorganisms responsible for severe diseases in man and animals. $C$. difficile infections are characterized by an intense inflammatory response while only modest inflammation occurs in $C$. sordellii infections. However, both pathogens produce closely related large clostridial glucosylating toxins that inactivate small GTPases. Michel Robert POPOFF (Paris, France) reported on the differential inactivation of Rho/Ras proteins by these Clostridium strains to explain the production or the prevention of an inflammatory response following infection (also reviewed by M-R. POPOFF in this issue).

Local tissue damage, characterized by edema, hemorrhage and necrosis, is a common consequence of viper bite envenoming. There is a growing effort to understand the contribution of inflammatory responses induced by the venom metalloproteinase, such as phospholipase $\mathrm{A}_{2}\left(\mathrm{PLA}_{2}\right)$, in the development of these lesions. Joseph KHOURY (Tripoli, Lebanon) documented enzymatic activities of the venom of the vipera Montivipera bornmuelleri, and exposed that the aqueous buds extract of Eucalyptus efficiently neutralized in vitro proteolytic, $\mathrm{PLA}_{2}$ and L-amino acid oxidase activities of $M$. bornmuelleri venom, depicting a promising natural source of antivenomic compounds against vipera species bites.

Five reports of new interesting results were emphasized in the last Miscellaneous session. The first two communications were in relation to the family of bacterial nucleotidyl cyclase toxins which, once secreted, exhibit potent virulence by their ability to invade eukaryotic cells. Hence, Alexandre CHENAL (Paris, France) studied the adenylate cyclase CyaA toxin secreted by Bordetella pertussis, the causative agent of whooping cough, which plays an essential role in the early stages of infection. He highlighted the involvement of calcium in several steps of the cell intoxication process by CyaA toxin including, in particular, the toxin folding into a stable, monomeric and functional form, and the translocation of its catalytic domain across the plasma membrane of the target cell (see the article by D.P. O'BRIEN $\boldsymbol{e t}$ al. in this issue). Alexander BELYY (Paris, France) identified the filamentous form of actin as the only eukaryotic cofactor necessary to specifically activate the adenylate cyclase ExoY toxin, secreted by the opportunistic human pathogen Pseudomonas aeruginosa (reviewed by A. BELYY et al. in this issue).

The following three reports were devoted to the latest research findings related to venom toxins. Finding new toxins is still a challenge in toxinology that must be met because many venom toxins have proved to be valuable pharmacological tools to investigate the functioning of diverse biological targets including ions channels, G-protein coupled receptors, transporters and enzymes. Hence, Naoual OUKKACHE (Casablanca, Morocco) detailed the purification, the in vivo toxicity, the complete amino acid sequence and the functional characterization of Am IT, a novel toxin isolated from the venom of the scorpion Androctonus mauretanicus. This toxin exhibits similar activity to toxins found in "Old World" and "New World" scorpion venoms. Laura DROCTOVÉ (Gif-sur-Yvette, France) presented new information on mambaquaretin-1, the first identified member of the mambaquaretin group of toxins from mamba venoms, which targets the vasopressin type 2 receptor primarily expressed in the kidney where it regulates fluid osmotic pressure and diuresis through the vasopressin hormone. She validated mambaquaretin-1 as a novel promising 
therapeutic candidate to treat kidney cysts, following convincing evidence of the toxin's ability to slow down the progression of polycystic kidney disease in a rodent model of this disease. Among the large diversity of three-finger toxins contained in the venoms of snakes belonging to the Elapidae family, some of them have the unique ability to interact selectively with muscarinic receptors. Justyna CIOLEK (Gif-sur-Yvette, France) had as objective to discover highly selective toxins of the muscarinic type-2 receptor, mostly expressed in the nervous system, and in the heart where it modulates pacemaker activity. She screened the venom of Dendroaspis polylepis black mamba on CHO cells stably expressing this type of receptor, and discovered a novel peptide, muscarinic toxin 9, able to selectively target and act as an inverse agonist of the muscarinic type-2 receptor.

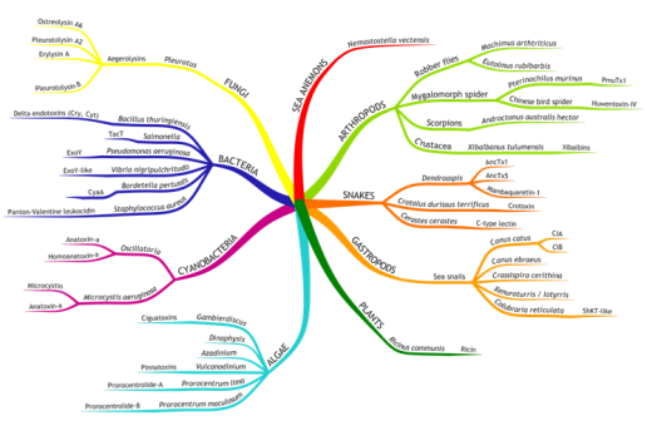

The first session of the $24^{\text {th }}$ SFET Meeting on Toxinology was devoted to Toxins and Evolution. From a general point of view, toxins may be seen as key adaptions to ensure evolutionary fitness and survival of a species, as exemplified by some toxins produced by Gram-positive, soil-dwelling bacteria and those found in robber fly and snake venoms. Hence, Alejandra BRAVO (Morelos, Mexico) improved our knowledge on how evolution of protein families produced by Bacillus thuringiensis (a Gram-positive, soil-dwelling bacterium, commonly used as a biological pesticide) creates such a diverse arsenal of toxins, mainly the Cry toxins, pointing out novel insights in the understanding of their mechanism of action, and in the molecular mechanism involved in the evolution of resistance to these toxins in some insects. Then, besides the morphological diversity of two gland systems, an interesting insight was provided by Stephan Holger DRUKEWITZ (Leipzig, Germany) into the venoms of the robber flies (Machimus arthtriticus and Eutolmus rufibarbis), whose toxins often evolved from proteins with an ancestral physiological function, were sub- or neo-functionalized during the evolution, and now act as toxins. This session ended with detailed information given by Wolfgang WUSTER (Bangor, UK) on the basis for understanding the selection pressures on snake venoms, which consists in an adequate knowledge of taxonomy and phylogeny, as well as rigorous natural history data on these animals and their interactions with prey and predators, and by Denis SERVENT (Gif-sur-Yvette, France) on the resurrection of ancestral proteins. This innovative approach exploits molecular evolution models to efficiently guide protein engineering, and thus generate novel functions, based on the well-known three-finger structural scaffold of the large diversity of toxins characterizing mamba venoms and displaying the unique property to interact with various subtypes of $\alpha$ adrenergic, dopaminergic and muscarinic receptors.

The second session, devoted to Toxins and Biodiversity, clearly illustrated the close correlation between the diversity of toxins and that of their biological targets. One of the most compelling outcomes of molecular microbiology attempts to understand bacterial behavior is the discovery of diverse toxin-antitoxin (TA) systems that control bacterial persistence. Persister cells, those cells tolerant to antibiotics, arise due to a state of dormancy induced by TA and are thought to be responsible for relapsing infections. TA systems are small genetic modules found on bacterial mobile genetic elements as well as in bacterial chromosomes, and consist of a stable toxin that disrupts an essential cellular process and a labile antitoxin that prevents toxicity. Sophie HELAINE (London, UK) investigated the activity of three of these TA toxins, bearing an acetyltransferase activity, from Salmonella. One well-known example is the diversity of compounds 
found in the venoms of the hyperdiverse group of Conoidea, including cone snails, i.e. small molecules, peptides and enzymes, largely dominated by that of disulfide-rich peptides, known as conotoxins. The evolution of the hyperdiverse group of Conoidea and their toxins was developed by Nicolas PUILLANDRE (Paris, France) who illustrated, with selected examples, some of the impacts of major rearrangements in the systematics of the group led by molecular phylogenies, both at the species level, with many cryptic species revealed, and at the phylogenetic level, with the recognition of many new family and genus-level lineages.

The diversity of toxins is evidenced by the constant discovery of novel peptides produced by various organisms, as exemplified below by those discovered from conoidean and non-conoidean neogastropods, as well as from Pancrustacea such as crustaceans and insects. Hence, Julien GIRIBALDI (Montpellier, France) reported the synthesis, structure determination, and biological activities of two new $\alpha$-conotoxins, CIA and CIB, discovered in the venom-gland transcriptome of the piscivorous species Conus catus. Consistent with their pharmacology, CIA likely participates in prey capture through muscle paralysis, while the putative role of CIB remains to be elucidated. The ShK toxin family, first discovered in the venom of sea anemones, is constituted of small peptides that specifically inhibit various types of voltage-dependent and calcium-activated potassium channels, and thus exert different biological functions including neurotoxic and immunosuppressive effects. Maria Vittoria MODICA (Napoli, Italy) presented new interesting information, based on molecular and phylogenetic analyses, concerning the ShK-like toxins produced by the Mediterranean vampire snail Colubraria reticulate. The complexity of protein and peptide cocktails expressed in the venom glands of the anchialine cave-dwelling remipede crustacean Xibalbanus tulumensis, appraised by proteomic and transcriptomic approaches, was further addressed by Björn Marcus VON REUMONT (London, UK). He confirmed the presence of peptide families in the venom of this crustacean, including novel peptide families named xibalbins. Finally, following the screening of a spider-venom library, Rosanna MARY (Montpellier, France) discovered a new peptide (PmuTx1) from the venom of the mygalomorph spider Pterinochilus murinus. This peptide is of great interest since it is the first one, identified so far, that fully blocks the current flowing through the T-type $\mathrm{Ca}_{\mathrm{v}} 3.2$ voltage-dependent calcium channel, a potential target for the development of new analgesic compounds.

The third session was dedicated to Toxins and Environment. To counteract the increasing risk of evolution of pest resistance to Bacillus thuringiensis $(B t)$, insecticidal proteins and their resulting reduced effectiveness were discussed. Due to the rapid and widespread adoption of transgenic crops developed with genes encoding these proteins, Lu LIU (Hayward, USA) highlighted the systematic evaluation of proteins from non- $B t$ microbes, as a new strategy, resulting in the identification of several new classes of insecticidal proteins for their activity spectra, in planta efficacy and potential mode of action.

The bright side of cyanobacteria, i.e. blue-green algae, and of the 5,000 species of marine micro-algae identified so far, is to transform planet earth's atmosphere to one suitable for aerobic metabolism and complex life. However, their dark side is to produce toxins, or compounds in the environment, that are detrimental to other organisms, including humans. Philipp HESS (Nantes, France) presented his recent efforts focused on the systematic mapping of these species, and related toxins and metabolites, as well as the consequences of their chemical diversity, exemplified by the dinoflagellate species Gambierdiscus and Dinophysis. Using a multidisciplinary approach, including bioinformatics, genomics, biochemistry, synthetic biology, molecular biology, analytical chemistry and structural elucidation, Rabia MAZMOUZ (Paris, France) reported new findings 
concerning the neurotoxic anatoxin-a, the cytotoxic cylindrospermopsin, and the hepatotoxic microcystin by studying the biosynthesis, function and regulation of these cyanotoxins. Little being known about venom production across a complex life cycle of animals, Yehu MORAN (Jerusalem, Israel) investigated the dynamics of venom production in the sea anemone Nematostella vectensis by various transcriptomic, transgenic and biochemical approaches, revealing that venom appears in all life stages of this species with a toxin composition that significantly changes between the developmental stages leading to an arsenal of toxin-producing cells that is much wider than expected.

Three reports of new interesting results were emphasized in the last Miscellaneous session. The first two communications were in relation to the family of bacterial nucleotidyl cyclase toxins that exhibit potent virulence via their ability to invade eukaryotic cells where they are stimulated by endogenous cofactors (calmodulin or actin) to produce large amounts of 3'5'-cyclic nucleoside monophosphate, in the continuity of the studies presented during the $23^{\text {rd }}$ SFET Meeting on Toxinology. Hence, Alexis VOEGELE (Paris, France) went on with the calmodulin-activatable adenylate cyclase CyaA toxin, secreted by Bordetella pertussis (the whooping cough agent), which is implicated in the early stages of infection. He highlighted the involvement of two arginine residues (R461 and R474) in the local membrane destabilization of eukaryotic cells, which facilitates toxin translocation. Alexander BELYY (Paris, France) identified a peptide segment that is crucial for the interactions between the actin-activatable adenylate cyclase ExoY toxin, secreted by Pseudomonas aeruginosa, and the filamentous form of actin, and between the actin-activatable adenylate cyclase ExoY-like toxin, secreted by Vibrio nigripulchritudo, and the monomeric form of actin.

Animal venoms can be seen as large natural libraries of biologically active molecules that are continuously refined by evolution, up to the point where every molecule is endowed with pharmacological properties that are potentially valuable in the context of human use and drug development. In this framework, Steve REYNAUD (Gif-sur-Yvette, France) was interested in the melanocortin-4-receptor, mainly expressed in the hypothalamus, where it plays a crucial role in energy homeostasis and satiety and whose deficiency is the first cause of monogenic obesity for which no specific treatment has been identified so far. The strategy that combined high-throughput transcriptomic and proteomic methods allowed the identification of 284 reliable peptide sequences, especially from small arthropods and cone snails, as ligands of the melanocortin-4-receptor. Thus, these innovative ligands are promising tools for pharmacological studies on this type of receptor.

We hope that this Toxicon Special Issue will be attractive and represent a comprehensive source of information for students and researchers in Toxinology.

\title{
Evelyne Benoit $^{1,2}$, Julien Barbier ${ }^{1}$ and Jordi Molgó ${ }^{1,2 *}$
}

${ }^{1}$ CEA, Institut des sciences du vivant Frédéric Joliot, Service d'Ingénierie Moléculaire des Protéines, Université Paris-Saclay,

${ }^{2}$ Institut des Neurosciences Paris-Saclay, UMR 9197 CNRS/Université Paris-Sud, Gif-sur-Yvette, France

\author{
*Corresponding author \\ E-mail address: jordi.molgo@cea.fr
}

